\title{
Effect of CCL11 on In Vitro Myogenesis and Its Clinical Relevance for Sarcopenia in Older Adults
}

\author{
Da Ae Kim ${ }^{1, *}$, So Jeong Park ${ }^{1, *}$, Jin Young Lee ${ }^{1}$, Jeoung Hee Kim², Seungjoo Lee ${ }^{2}$, Eunju Lee ${ }^{3}$, Il-Young Jang ${ }^{3}$, \\ Hee-Won Jung 3 , Jin Hoon Park ${ }^{2}$, Beom-Jun Kim ${ }^{4}$ \\ ${ }^{1}$ Asan Institute for Life Sciences, ${ }^{2}$ Department of Neurological Surgery, Divisions of ${ }^{3}$ Geriatrics, ${ }^{4}$ Endocrinology and \\ Metabolism, Department of Internal Medicine, Asan Medical Center, University of Ulsan College of Medicine, Seoul, Korea
}

Background: The C-C motif chemokine ligand 11 (CCL11) has been receiving attention as a potential pro-aging factor. Accordingly, it may be involved in muscle metabolism and sarcopenia, a key component of aging phenotypes. To clarify this potential, we investigated the effects of CCL11 on in vitro muscle biology and its clinical relevance for sarcopenia parameters in older adults.

Methods: Myogenesis was induced in mouse $\mathrm{C} 2 \mathrm{C} 12$ myoblasts with $2 \%$ horse serum. Human blood samples were collected from 79 participants who underwent a functional assessment. Thereafter, CCL11 level was measured using a quantikine ELISA kit. Sarcopenia was defined using the Asian-specific guideline.

Results: Recombinant CCL11 treatment significantly stimulated myogenesis in a dose-dependent manner, and consistently increased the expression of myogenic differentiation markers. Among the C-C chemokine receptors (CCRs), CCR5, not CCR2 and CCR3, was predominantly expressed in muscle cells. Further, the CCR5 inhibitor blocked recombinant CCL11-stimulated myogenesis. In a clinical study, serum CCL11 level was not significantly different according to the status of sarcopenia, low muscle mass, weak muscle strength, and poor physical performance, and was not associated with skeletal muscle index, grip strength, short physical performance battery score, gait speed, and time to complete 5 chair stands, after adjusting for sex, age, and body mass index.

Conclusion: Contrary to expectations, CCL11 exerted beneficial effects on muscle metabolism at least in vitro system. However, its impact on human muscle health was not evident, suggesting that circulating CCL11 may not be a useful biomarker for sarcopenia risk assessment in older adults.

Keywords: Chemokine CCL11; Muscle development; Sarcopenia; Aging; Biomarkers

\section{INTRODUCTION}

Aging is characterized by a universal, progressive time-depen-

Received: 9 December 2020, Revised: 23 January 2021,

Accepted: 22 February 2021

Corresponding authors: Jin Hoon Park

Department of Neurological Surgery, Asan Medical Center, University of Ulsan College of Medicine, 88 Olympic-ro 43-gil, Songpa-gu, Seoul 05505, Korea Tel: +82-2-3010-3553, Fax: +82-2-2045-4123, E-mail: jhpark@amc.seoul.kr

Beom-Jun Kim

Division of Endocrinology and Metabolism, Department of Internal Medicine, Asan Medical Center, University of Ulsan College of Medicine, 88 Olympic-ro 43-gil, Songpa-gu, Seoul 05505, Korea

Tel: +82-2-3010-5876, Fax: +82-2-3010-6962, E-mail: umkbj0825@amc.seoul.kr dent functional decline that occurs across multiple organ systems, resulting in increased vulnerability to death $[1,2]$. This multi-step deterioration represents the main risk factor for sev-

\section{Copyright $\odot 2021$ Korean Endocrine Society}

This is an Open Access article distributed under the terms of the Creative Commons Attribution Non-Commercial License (https://creativecommons.org/ licenses/by-nc/4.0/) which permits unrestricted non-commercial use, distribution, and reproduction in any medium, provided the original work is properly cited.

\footnotetext{
*These authors contributed equally to this work.
} 
eral debilitating pathologies, including cardiovascular diseases, cancer, neurodegeneration, and osteoporosis $[3,4]$. Importantly, the increased number of adults in the elderly population and improvements in healthcare and sanitary conditions have led to worldwide interest in the discovery of effective measures to slow or reverse the aging process. Although several contributors, such as cellular senescence, oxidative stress, and dysregulation of energy homeostasis, are known to be involved in ageassociated physiologic changes $[5,6]$, heterochronic parabiosis experiments have highlighted the critical role of circulating factors in the aging process [7-9]. In detail, the exposure of old mice to young blood rejuvenated aged organs and tissues $[10,11]$, whereas an exchange with old blood rapidly inhibited the physiologic functions of young mice [12]. Based on these backgrounds, researchers in this field continue to dedicate their efforts to identifying pro-youthful or pro-aging factors in blood $[9,13]$.

Sarcopenia corresponds to a gradual and generalized skeletal muscle disorder that involves the accelerated loss of muscle mass and function [14-16]. Among the multifactorial causes, cellular and molecular changes during aging contribute to an imbalance between the anabolic and catabolic pathways of muscle protein, and this condition commonly occurs in older people [17]. Sarcopenia is thus considered to be a key component of aging phenotypes. Although sarcopenia has long been considered as an unavoidable outcome of aging, the perception that it should be overcome has been increased due to advances in knowledge (i.e., skeletal muscle homeostasis might be reversible) $[18,19]$. Importantly, sarcopenia is strongly associated with increased vulnerability to falls, frailty, and death [17,20]. Consequently, the identification of factors affecting muscle metabolism has become the focus of intense research to extend health span in old age.

C C motif chemokine ligand 11 (CCL11), also denoted as eotaxin-1, exerts biologic effects via $\mathrm{C}-\mathrm{C}$ chemokine receptors (CCRs) [21]. CCL11 is well known as a potent chemoattractant for eosinophils and is thus mainly implicated in allergic responses [22,23]. Accumulating evidence indicates that CCL11 may also play a pivotal role in age-related degeneration. Comparison of young isochronic and heterochronic cohorts revealed that plasma CCL11 level was markedly higher in heterochronic parabionts [8]. Consistently, a positive correlation was found between circulating CCL11 concentration and chronological age in both mice and humans, and systemic CCL11 injection decreased neurogenesis and cognitive performance in young mice [8]. Further, exogenous CCL11 disturbed bone remodeling by stimulating preosteoclast migration and resultant bone resorption [24]. In addition to the secreted feature of CCL11, these results suggest that this protein could be a potential systemic pro-aging factor and could be involved in muscle metabolism and age-associated sarcopenia. To clarify this possibility, we examined the effects of CCL11 on in vitro muscle biology and its clinical relevance for sarcopenia parameters in older adults.

\section{METHODS}

\section{Cell culture and reagents}

Mouse C2C12 myoblasts were purchased from the American Type Culture Collection (ATCC, Manassas, VA, USA) and maintained in Dulbecco's Modified Eagle's medium (DMEM) supplemented with $10 \%$ fetal bovine serum, $20 \mathrm{mM}$ hydroxyethyl piperazine ethane sulfonic acid, $2 \mathrm{mM}$ L-glutamine, 100 $\mathrm{U} / \mathrm{mL}$ penicillin, and $0.1 \mathrm{mg} / \mathrm{mL}$ streptomycin (Life Technologies Corp., Carlsbad, CA, USA) at $37^{\circ} \mathrm{C}$ in a humidified atmosphere containing $5 \% \mathrm{CO}_{2}$. To induce myogenesis, cells were grown to $90 \%$ confluency in maintenance media and switched to differentiation media (DMEM with $2 \%$ horse serum) for 3 or 4 days of culture. Recombinant CCL11 was purchased from R\&D Systems (cat. no. 420-ME-100; Minneapolis, MN, USA).

\section{Immunofluorescence}

Differentiated C2C12 cells were fixed in 4\% paraformaldehyde for 15 minutes, washed twice with phosphate buffered saline (PBS), permeabilized in $0.01 \mathrm{M}$ sodium citrate buffer containing $0.1 \%$ Triton X-100 for 10 minutes, and washed twice with PBS. The cells were blocked with $2 \%$ bovine serum albumin (BSA) in PBS for 1 hour and subsequently incubated with antimyosin heavy chain (MyHC) antibody (MF20; Developmental Studies Hybridoma Bank, Iowa City, IA, USA) at $4^{\circ} \mathrm{C}$ overnight. Cells were incubated with Alexa Flour 555-conjugated secondary antibodies (Cell Signaling, Danvers, MA, USA; $1: 1,000$ dilution) for 1 hour and washed with PBST $(0.2 \%$ Tween-20 in PBS). Thereafter, they were incubated with 4,6-diamidino-2-phenyindole (DAPI; Sigma-Aldrich, St. Louis, MO, USA; 1:10,000 dilution) for 2 minutes and washed with PBS. The samples were mounted using Fluoromount G (Southern Biotech, Birmingham, AL, USA) and images were captured using a fluorescence microscope (Carl Zeiss, Jena, Germany). The area of the MyHC-stained myotubes was calculated using the ZEN 2 (blue edition) software (Carl Zeiss). Fusion index (\%) was calculated using the following equation: $100 \times$ number of 
nuclei in $\mathrm{MyHC}^{+}$myotubes per total number of nuclei in $\mathrm{MyHC}^{+}$myocytes and myotubes.

\section{Western blot analysis}

Cells were lysed in radioimmunoprecipitation assay (RIPA) buffer (50 mM Tris- $\mathrm{HCl}$ [pH 7.4], $150 \mathrm{mM} \mathrm{NaCl,} \mathrm{1 \%} \mathrm{Triton}$ X-100, $1 \mathrm{mM}$ ethylenediaminetetraacetic acid, $1 \mathrm{mM}$ ethylene glycol tetraacetic acid, $0.1 \%$ sodium dodecyl sulfate [SDS], 1\% sodium deoxycholate, $1 \mathrm{mM} \mathrm{Na} \mathrm{VO}_{4}, 1 \mathrm{mM}$ sodium fluoride, 1 $\mathrm{mM}$ phenylmethylsulfonyl fluoride, and protease inhibitor cocktail). After a 30-minute incubation on ice, the lysates were centrifuged at $14,000 \mathrm{rpm}$ for 20 minutes at $4^{\circ} \mathrm{C}$. The protein concentration was measured with a bicinchoninic Acid (BCA) protein assay kit (Pierce Chemical Co., Rockford, IL, USA). Protein samples were separated by SDS-polyacrylamide gel electrophoresis (PAGE), transferred to a polyvinylidene fluoride membrane, and immunoblotted with antibodies [25]. The primary antibodies were: MyHC (MF20); myogenin (sc-12732) and CCR5 (sc-178933) (Santa Cruz Biotechnology, Dallas, TX, USA); and $\beta$-tubulin (T2200; Sigma-Aldrich).

\section{Quantitative reverse-transcription polymerase chain reaction}

Total RNA was extracted using TRIzol reagent (Invitrogen, Carlsbad, CA, USA) according to the manufacturer's protocol. After first-strand cDNA synthesis with the Superscript III FirstStrand Synthesis System (Invitrogen) using oligo dT primers, quantitative reverse-transcription polymerase chain reaction was performed in triplicate on a Light Cycler 480 SYBR Green I Master (Roche, Mannheim, Germany) [26]. The primers for myogenin (NM_031189.2) and MyHC (NM_001013397.2) were obtained from Applied Biosystems (Foster City, CA, USA). The threshold cycle ( $\mathrm{Ct}$ ) value for each gene was normalized to the $\mathrm{Ct}$ value of the 18S rRNA (NR_003278.3).

\section{Ligand and receptor binding assay}

Recombinant CCL11 was coated onto the wells of maxisorp 96well microtiter plates and incubated for 18 hours at $4^{\circ} \mathrm{C}$. Each well was washed three times with PBST $(0.2 \%$ Tween-20 in PBS). Thereafter, the plates were blocked with 2\% BSA in PBST for 2 hours. Cell lysates were added to the plates and incubated for 2 hours. The wells were then washed three times [27]. Preparations of the CCR5 antibody (Santa Cruz Biotechnology) in blocking solution were added to the plates and allowed to react for 2 hours. After washing, horseradish peroxidase (HRP)-linked antibody (Cell signaling Technology) was added and the lysates were incubated for 2 hours; this was followed by five rounds of washes. The reaction was developed with $100 \mu \mathrm{L}$ tetramethylbenzidine (TMB) substrate solution and stopped with $100 \mu \mathrm{L}$ of $1 \mathrm{~N} \mathrm{H}_{2} \mathrm{SO}_{4}$. The absorbance in the microtiter plates was measured at $450 \mathrm{~nm}$ using a microplate reader (Infinite 200 PRO; Tecan Life Sciences, Zürich, Switzerland).

\section{Study participants}

We consecutively recruited Koreans who visited the Division of Geriatrics, Department of Internal Medicine, Asan Medical Center (AMC, Seoul, Korea) to undergo a comprehensive geriatric assessment between July 2019 and April 2020 [28]. Participants whose life expectancy was expected to be less than 1 year owing to end-stage renal failure, symptomatic heart failure, or malignancy were excluded. After the exclusion of ineligible participants, blood samples were collected from 79 eligible participants with their consent. This study was approved by the AMC Institutional Review Board (no. 2020-0259). Written informed consent was obtained from all enrolled participants.

\section{Functional status and sarcopenia assessment}

Experienced nurses were charged with collecting participants' information, such as demographic characteristics and surgical or medical histories, through detailed interviews and reviews of medical records. Handgrip strength of the dominant side was measured using Jamar hydraulic hand dynamometer (Patterson Medical, Warrenville, IL, USA) [29]. Participants were instructed to sit comfortably, bend their elbow at 90 degrees, and hold the dynamometer as tightly as possible. The maximum value was adapted after all tests were conducted twice at intervals of 1 minute or more. Time to complete 5 chair stands (s) and usual gait speed $(\mathrm{m} / \mathrm{sec})$ from a 4-m walk were assessed [30]. The short physical performance battery (SPPB) consists of gait speed, standing balance, and repeated chair stands [31]. In the standing balance test, including tandem stance, semi-tandem stance, and side-by-side stance, the participants were instructed to stand for up to 10 seconds. Ranging from 0 to 12 points, a higher SPPB score indicates better lower extremity function.

A bioelectrical impedance analyzer (InBody S10; InBody, Seoul, Korea) with measuring frequencies of 1, 5, 50, 250, 500, and $1,000 \mathrm{kHz}$ was used to evaluate the body compositions, including muscle mass (whole body lean body mass minus bone mineral content) [32]. Appendicular skeletal muscle mass (ASM) was defined as the sum of the muscle mass of both arms and legs. Skeletal muscle mass index (SMI) was calculated by adjusting the 
ASM to the height squared to achieve an objective comparison of muscle mass between participants [33]. Lastly, sarcopenia was classified according to the 2019 Consensus Guidelines from the Asian Working Group for Sarcopenia [34]. Briefly, older patients with low muscle mass (SMI $<7.0 \mathrm{~kg} / \mathrm{m}^{2}$ in men, $<5.7 \mathrm{~kg} / \mathrm{m}^{2}$ in women), weak muscle strength (handgrip strength $<28 \mathrm{~kg}$ in men, $<18 \mathrm{~kg}$ in women), and/or poor physical performance (5time chair stand test $\geq 12$ seconds, gait speed $<1.0 \mathrm{~m} / \mathrm{sec}$, or SPPB score $\leq 9$ points) were diagnosed with sarcopenia.

\section{Measurement of CCL11 in human serum}

Blood samples were collected from the antecubital vein of each participant. After sample centrifugation at 3,000 rpm for $5 \mathrm{~min}-$ utes at $4^{\circ} \mathrm{C}$, the supernatants were carefully collected to exclude cell components. All samples that displayed clotting or hemolysis were discarded. Prior to determining the serum concentrations, the serum samples were stored at $-80^{\circ} \mathrm{C}$. Serum CCL11 level was measured using a quantikine enzyme-linked immunosorbent assay (ELISA) kit (Cat. No. DTX00; R\&D Systems) in accordance with the manufacturer's instructions. The lower limit of detection of the kit was $5 \mathrm{pg} / \mathrm{mL}$, and the intra- and interassay coefficients of variation were less than $5.3 \%$ and $11.5 \%$, respectively.

\section{Statistical analysis}

The in vitro data are expressed as mean \pm standard error of mean of at least three independent experiments conducted with triplicate measurements, unless otherwise specified. The MannWhitney $U$ test and the analysis of variance (ANOVA) with post hoc analysis via Tukey's honest significance test were used to evaluate the significance of the differences between two groups and between $\geq 3$ groups, respectively.

The clinical data are presented as mean \pm standard deviation or numbers and percentages, unless otherwise specified. The Student's $t$ tests for continuous variables and chi-square tests for categorical variables were used to compare the baseline characteristics of the study participants according to sarcopenia status. Kolmogorov-Smirnov testing revealed that serum CCL11 concentrations were normally distributed $(P=0.302)$. The relationship between age and serum CCL11 level was investigated using Pearson correlation analysis. Before and after adjustments for sex, age, and body mass index (BMI), analysis of covariance (ANCOVA) was used to generate and compare the estimated means with 95\% confidence intervals (CIs) in serum CCL11 level according to the status of sarcopenia and the related parameters. The association between serum CCL11 level and sar- copenia parameters was examined using a linear regression analysis. ANCOVA was also used to generate and compare the estimated means with 95\% CIs in sarcopenia parameters according to the serum CCL11 quartiles. All statistical analyses were performed using the SPSS version 18.0 (SPSS Inc., Chicago, IL, USA). $P<0.05$ was considered statistically significant.

\section{RESULTS}

\section{In vitro effects of recombinant CCL11 on muscle differentiation}

To determine the potential role of CCL11 in muscle biology, we treated $\mathrm{C} 2 \mathrm{C} 12$ myoblasts with recombinant CCL11 in vitro. While recombinant CCL11 did not affect proliferation or viability of myoblasts (data not shown), it significantly increased myotube area, myotube area per myotube, nuclei number per myotube, and fusion index in a dose-dependent manner, thereby revealing the CCL11-stimulated myogenesis (Fig. 1A). Consistently, the protein and mRNA expression levels of the myogenic differentiation markers, such as myogenin and MyHC, were markedly increased by recombinant CCL11 (Fig. 1B, C, respectively).

Among the potential receptors mediating the action of CCL11 on different types of cells $[35,36]$, only CCR5 (i.e., not CCR2 or CCR3) was strongly expressed in both myoblasts and myotubes (Fig. 2A). A binding affinity experiment using ELISA also showed that the amount of CCL11-associated CCR5 increased as the amount of $\mathrm{C} 2 \mathrm{C} 12$ myoblast lysate increased (Fig. 2B), indicating that CCL11 directly interacts with CCR5 in muscle cells. Aligning with these results, treating $\mathrm{C} 2 \mathrm{C} 12$ myoblasts with CCR5 inhibitor completely attenuated recombinant CCL11-induced myogenesis (Fig. 3C). Such findings suggest that CCR5 mediates the effects of CCL11 on myogenic differentiation.

\section{Association between circulating CCL11 level and sarcopenia parameters in humans}

To investigate the clinical implication of in vitro results in agingassociated sarcopenia, we measured CCL11 level in the blood of 79 older adults. The baseline characteristics of the adults are presented in Table 1 . Of the 12 cases with sarcopenia and 67 controls without sarcopenia, nine (75.0\%) and 34 (50.7\%) were women, respectively. The mean ages of cases and controls were $72.1 \pm 4.3$ and $68.8 \pm 6.4$, respectively. Participants with sarcopenia had lower weight, height, BMI, ASM, SMI, grip strength, and gait speed than those without sarcopenia. However, there were no significant differences in terms of SPPB score and time 
Control

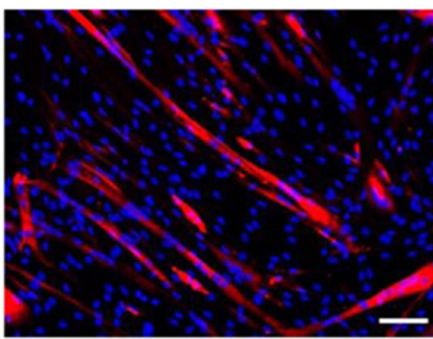

CCL11 $1 \mathrm{nM}$
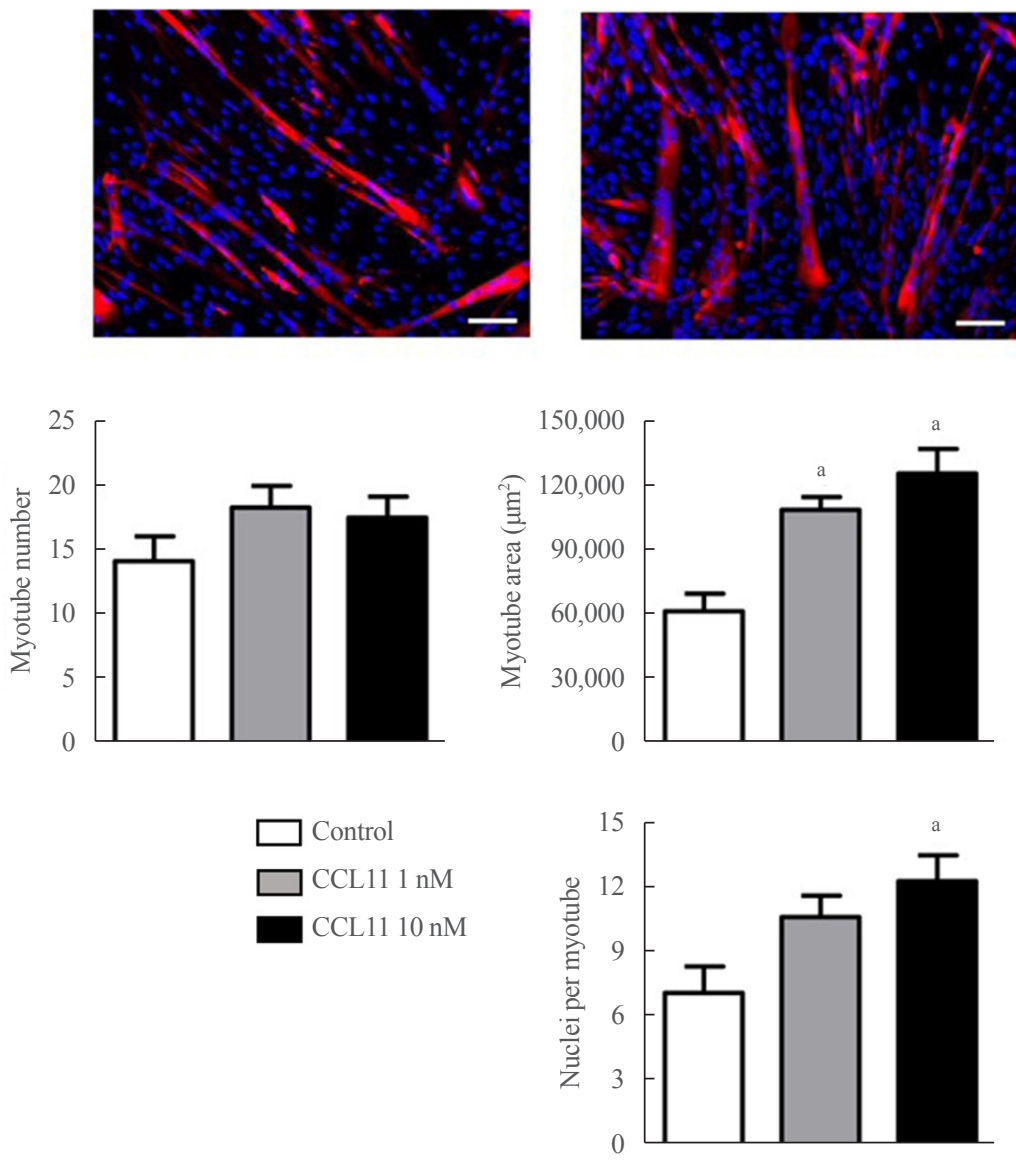

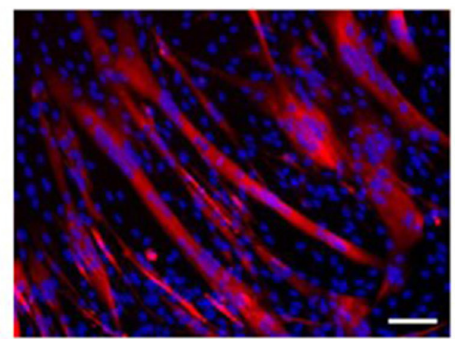

CCL11 $10 \mathrm{nM}$
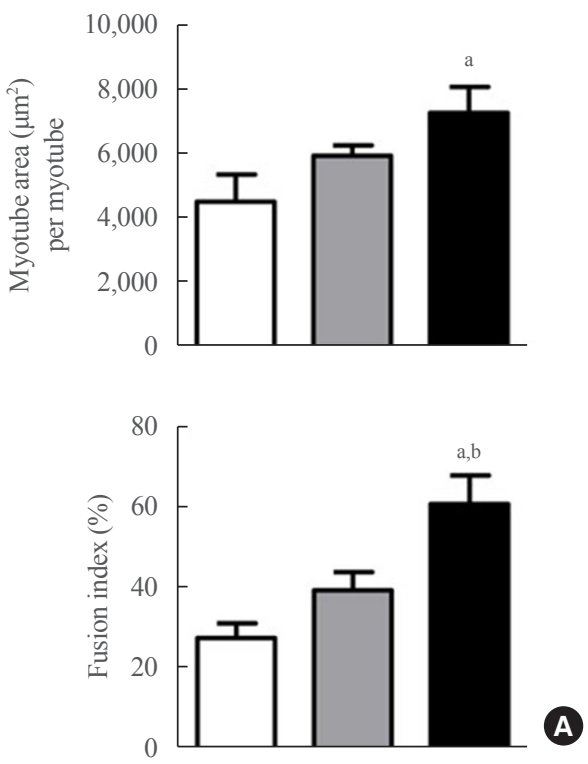

$\mathrm{MB} \frac{\mathrm{MT}}{-+} \quad \mathrm{MB} \frac{\mathrm{MT}}{-+}$

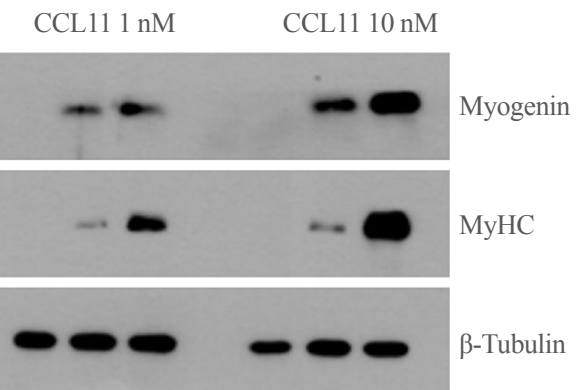

B

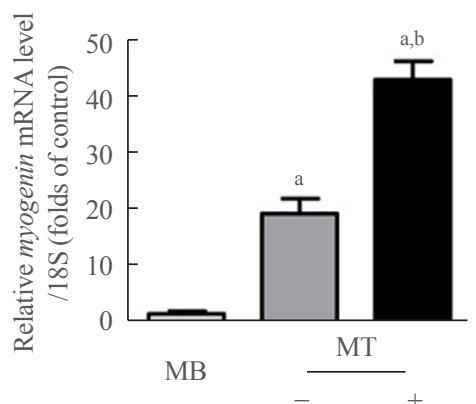

CCL11 1 nM

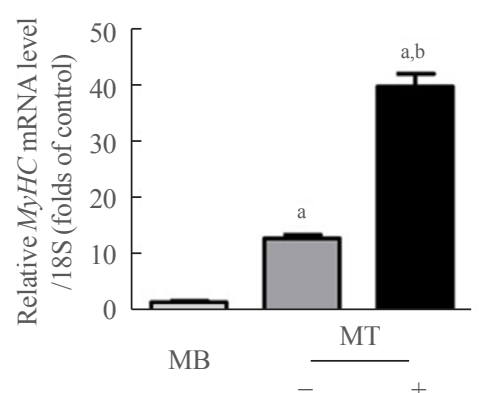

CCL11 $10 \mathrm{nM}$

Fig. 1. Recombinant C-C motif chemokine ligand 11 (CCL11) stimulates in vitro myogenesis. (A) Mouse C2C12 myoblasts (MBs) were differentiated into myotubes (MTs) with $2 \%$ horse serum after exposure to the indicated concentrations of recombinant CCL11 for 4 days. MTs were stained with the anti-myosin heavy chain (MyHC) antibody while the nuclei were counterstained with 4,6-diamidino-2-phenyindole (DAPI). Quantitative results per field are presented. Scale bars, $100 \mu \mathrm{m}$. (B) Western blot and (C) quantitative reverse-transcription polymerase chain reaction analyses of myogenin and $\mathrm{MyHC}$ in $\mathrm{C} 2 \mathrm{C} 12$ cells without or with $2 \%$ horse serum in the presence of the indicated concentrations of recombinant CCL11 for 3 days, respectively. Data are expressed as mean \pm standard error of mean. ${ }^{a} P<0.05$ vs. control or $\mathrm{MB} ;{ }^{\mathrm{b}} \mathrm{P}<0.05$ vs. CCL11 $1 \mathrm{nM}$ or untreated MT. 


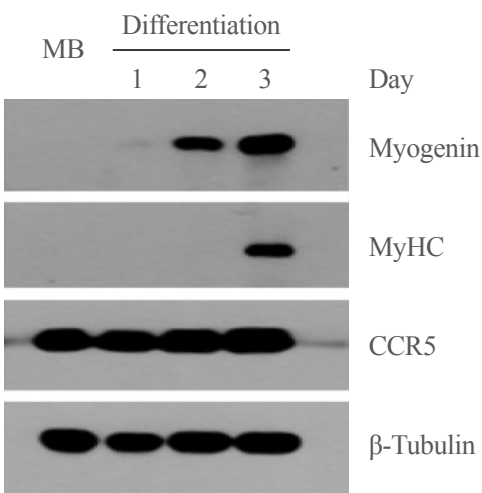

Control
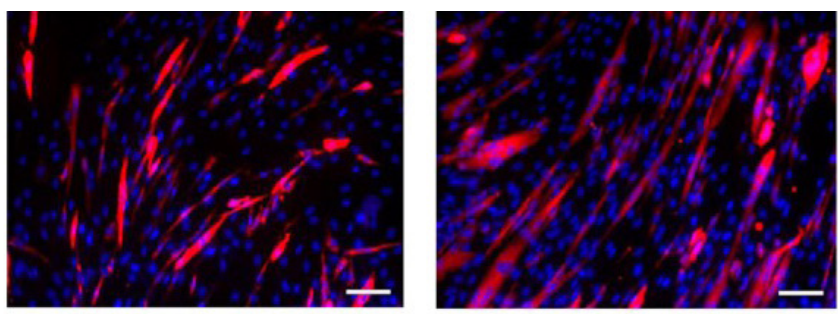

CCR5

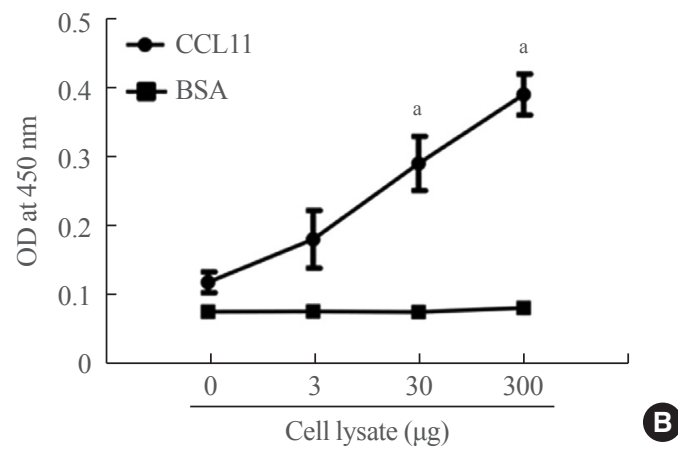

CCL11 $10 \mathrm{nM+}$

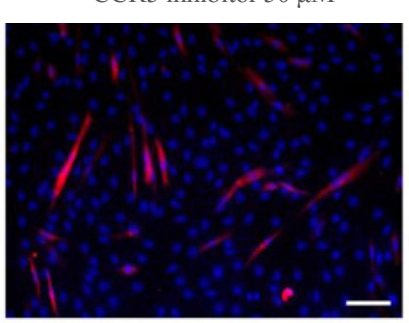

CCR5 inhibitor $50 \mu \mathrm{M}$

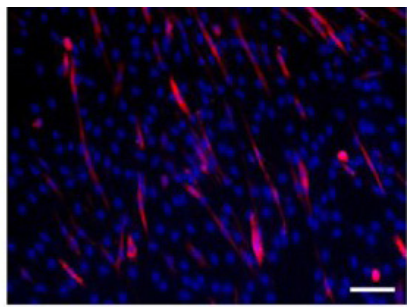

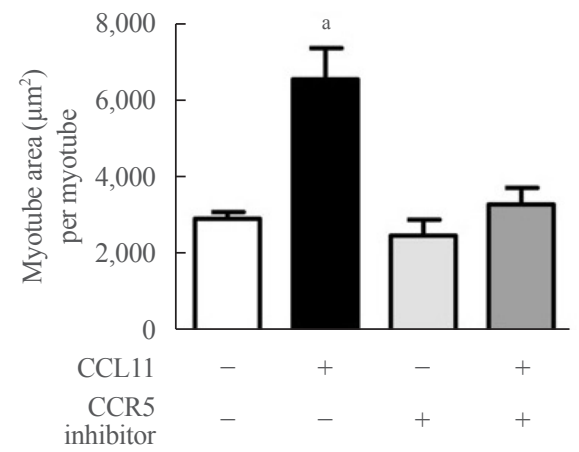
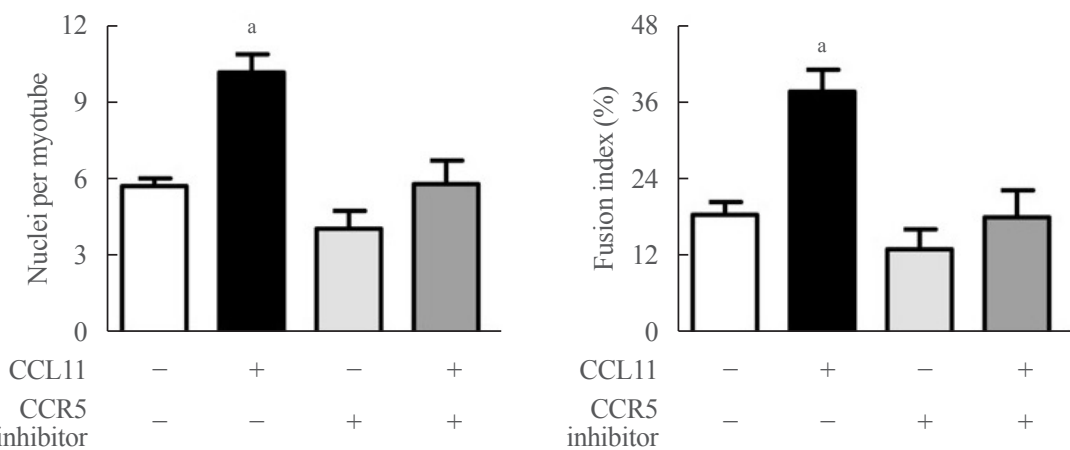

C

Fig. 2. C-C chemokine receptor 5 (CCR5) is the major receptor for C-C motif chemokine ligand 11 (CCL11) in muscle cells. (A) Mouse $\mathrm{C} 2 \mathrm{C} 12$ myoblasts (MBs) were differentiated into myotubes with $2 \%$ horse serum for the indicated days. Western blot analysis was performed to determine the expression of myogenin, myosin heavy chain (MyHC), and CCR5. (B) Interaction between CCL11 and CCR5 in C2C12 MBs using binding enzyme-linked immunosorbent assay (ELISA). Varying amounts of cell lysates were incubated in CCL11- or bovine serum albumin (BSA)-coated wells. Thereafter, the level of CCR5 was determined by ELISA. (C) Mouse C2C12 MBs were differentiated into myotubes with $2 \%$ horse serum in the presence or absence of recombinant CCL11 and/or CCR5 inhibitor for 4 days. Myotubes were stained with the anti-MyHC antibody while the nuclei were counterstained with 4,6-diamidino-2-phenyindole (DAPI). Quantitative results per field are presented. Scale bars, $100 \mu \mathrm{m}$. Data are expressed as mean \pm standard error of mean. OD, optical density. ${ }^{\mathrm{a}} P<0.05$ vs. control.

to complete 5 chair stands between the two groups.

Pearson correlation analyses with scatter plots revealed that age was positively correlated with serum CCL11 level (Supplemental Fig. S1).

Differences in serum CCL11 level according to the status of sarcopenia and related parameters were assessed using ANCOVA (Fig. 3). Before and after controlling for potential confounders, such as sex, age, and BMI, serum CCL11 concentrations were not significantly different between participants with and without sarcopenia, low muscle mass, weak muscle strength, and poor physical performance.

Linear regression analyses were adopted to investigate the association between serum CCL11 level and sarcopenia parameters (Table 2). However, serum CLL11 levels did not correlate with SMI, grip strength, SPPB score, gait speed, and time to complete 5 chair stands, regardless of the adjustment models. 

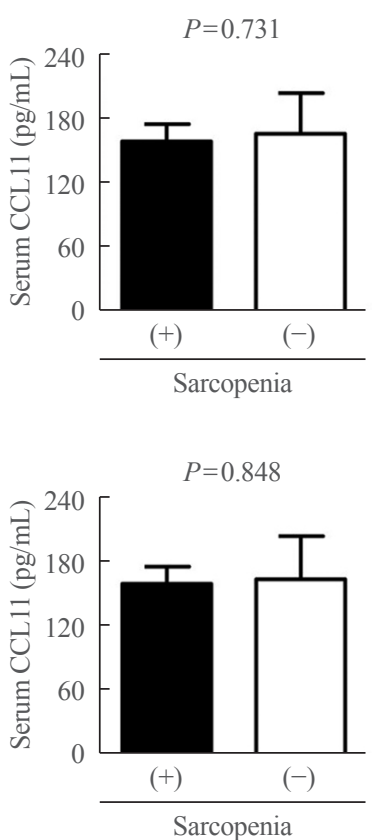
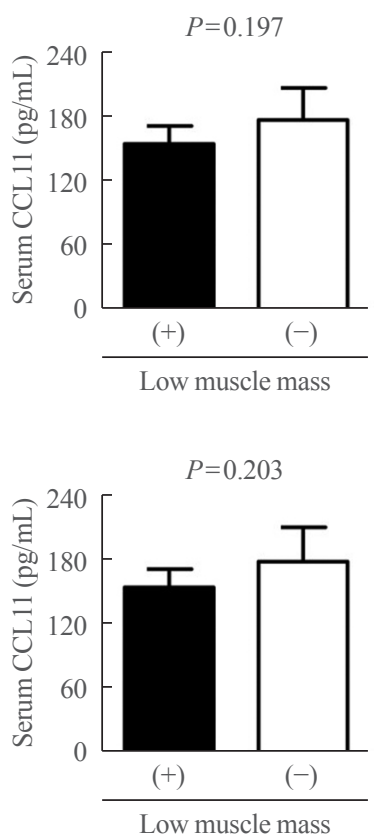
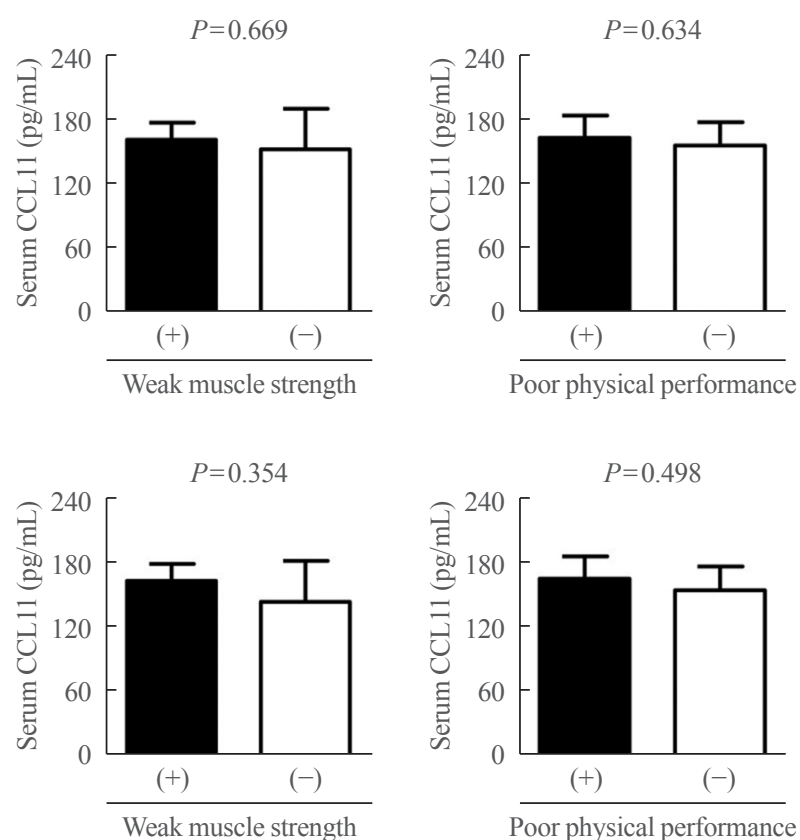

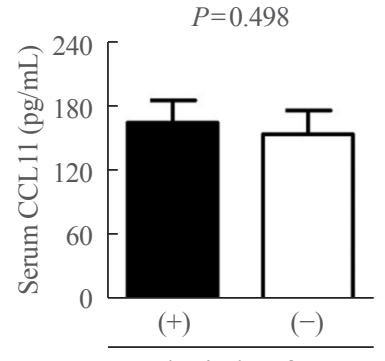

$\overline{\text { Poor physical performance }}$

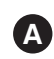

B

Fig. 3. Differences in serum C-C motif chemokine ligand 11 (CCL11) levels based on the status of sarcopenia and related parameters (A) before and (B) after adjusting for sex, age, and body mass index (BMI). Analysis of covariance was used to generate and compare the estimated means with $95 \%$ confidence intervals.

Table 1. Baseline Characteristics of the Study Participants According to Their Sarcopenia Status

\begin{tabular}{lccc}
\hline Variable & $\begin{array}{c}\text { Sarcopenia } \\
(n=12)\end{array}$ & $\begin{array}{c}\text { No sarcopenia } \\
(n=67)\end{array}$ & $P$ value \\
\hline Sex & & & 0.120 \\
$\quad$ Male & $3(25.0)$ & $33(49.3)$ & \\
$\quad$ Female & $9(75.0)$ & $34(50.7)$ & \\
Age, yr & $72.1 \pm 4.3$ & $68.8 \pm 6.4$ & 0.094 \\
Weight, kg & $59.4 \pm 9.3^{\mathrm{a}}$ & $69.4 \pm 10.1^{\mathrm{a}}$ & $0.002^{\mathrm{a}}$ \\
Height, cm & $155.6 \pm 2.9^{\mathrm{a}}$ & $160.0 \pm 9.3^{\mathrm{a}}$ & $0.003^{\mathrm{a}}$ \\
Body mass index, $\mathrm{kg} / \mathrm{m}^{2}$ & $24.5 \pm 3.8^{\mathrm{a}}$ & $27.1 \pm 2.9^{\mathrm{a}}$ & $0.010^{\mathrm{a}}$ \\
\hline ASM, kg & $12.8 \pm 1.2^{\mathrm{a}}$ & $18.5 \pm 4.8^{\mathrm{a}}$ & $<0.001^{\mathrm{a}}$ \\
SMI, kg/m ${ }^{2}$ & $5.28 \pm 0.48^{\mathrm{a}}$ & $7.10 \pm 1.15^{\mathrm{a}}$ & $<0.001^{\mathrm{a}}$ \\
Grip strength, kg & $22.8 \pm 7.1^{\mathrm{a}}$ & $29.2 \pm 8.9^{\mathrm{a}}$ & $0.022^{\mathrm{a}}$ \\
\hline SPPB score (range, 0-12) & $9.0 \pm 3.1$ & $10.9 \pm 1.6$ & 0.062 \\
Gait speed, m/sec & $0.74 \pm 0.31^{\mathrm{a}}$ & $1.09 \pm 0.29^{\mathrm{a}}$ & $<0.001^{\mathrm{a}}$ \\
Chair stand, sec & $16.7 \pm 14.9$ & $10.3 \pm 4.3$ & 0.172 \\
\hline
\end{tabular}

Values are expressed as number (\%) or mean \pm standard deviation. The Student's $t$-tests for continuous variables and chi-square tests for categorical variables were employed for comparisons between two groups. ASM, appendicular skeletal muscle mass; SMI, skeletal muscle mass index; SPPB, short physical performance battery.

${ }^{\text {a }}$ Statistically significant values.
To determine whether the correlation between serum CCL11 levels and sarcopenia parameters might exhibit a threshold effect rather than a gradual effect, the participants were divided into quartile groups according to serum CCL11 levels (Fig. 4). However, there was no significant difference in SMI, grip strength, SPPB score, gait speed, and time to complete 5 chair stands among the quartile groups, before and after adjusting for the confounders.

\section{DISCUSSION}

Based on backgrounds that implicated CCL11 in diverse agerelated diseases, we performed experiments to observe CCL11induced myogenesis. However, subsequent investigation to understand its clinical implications revealed no correlation between circulating CCL1 levels and muscle phenotypes in older adults. These findings indicate that the effects of CCL11 on in vitro muscle biology might not translate to humans, and that blood CCL11 level might not be a useful biomarker for sarcopenia risk assessment.

As one of efforts to find out the possible contributors explaining the development of sarcopenia, we have been particularly interested in the role of CCL11 on muscle metabolism. Previous studies reported that exogenous CCL11 treatment impaired neu- 
Table 2. Linear Regression Analyses to Determine the Association between Serum CCL11 Level and Sarcopenia Parameters

\begin{tabular}{|c|c|c|c|c|c|c|c|c|}
\hline & \multicolumn{4}{|c|}{ Unadjusted } & \multicolumn{4}{|c|}{ Sex, age, and BMI adjusted } \\
\hline & Unstandardized $\beta$ & SE & Standardized $\beta$ & $P$ value & Unstandardized $\beta$ & SE & Standardized $\beta$ & $P$ value \\
\hline SMI & -0.002 & 0.002 & -0.086 & 0.449 & -0.002 & 0.001 & -0.120 & 0.116 \\
\hline Grip strength & -0.017 & 0.015 & -0.123 & 0.280 & -0.015 & 0.010 & -0.107 & 0.133 \\
\hline SPPB score & -0.003 & 0.004 & -0.103 & 0.369 & -0.002 & 0.004 & -0.064 & 0.582 \\
\hline Gait speed & 0.000 & 0.001 & 0.001 & 0.994 & 0.000 & 0.001 & 0.034 & 0.761 \\
\hline Chair stand & 0.003 & 0.013 & 0.030 & 0.797 & -0.001 & 0.013 & -0.012 & 0.918 \\
\hline
\end{tabular}

The Enter method was applied to this model.

CCL11, C-C motif chemokine ligand 11; BMI, body mass index; SE, standard error; SMI, skeletal muscle mass index; SPPB, short physical performance battery.
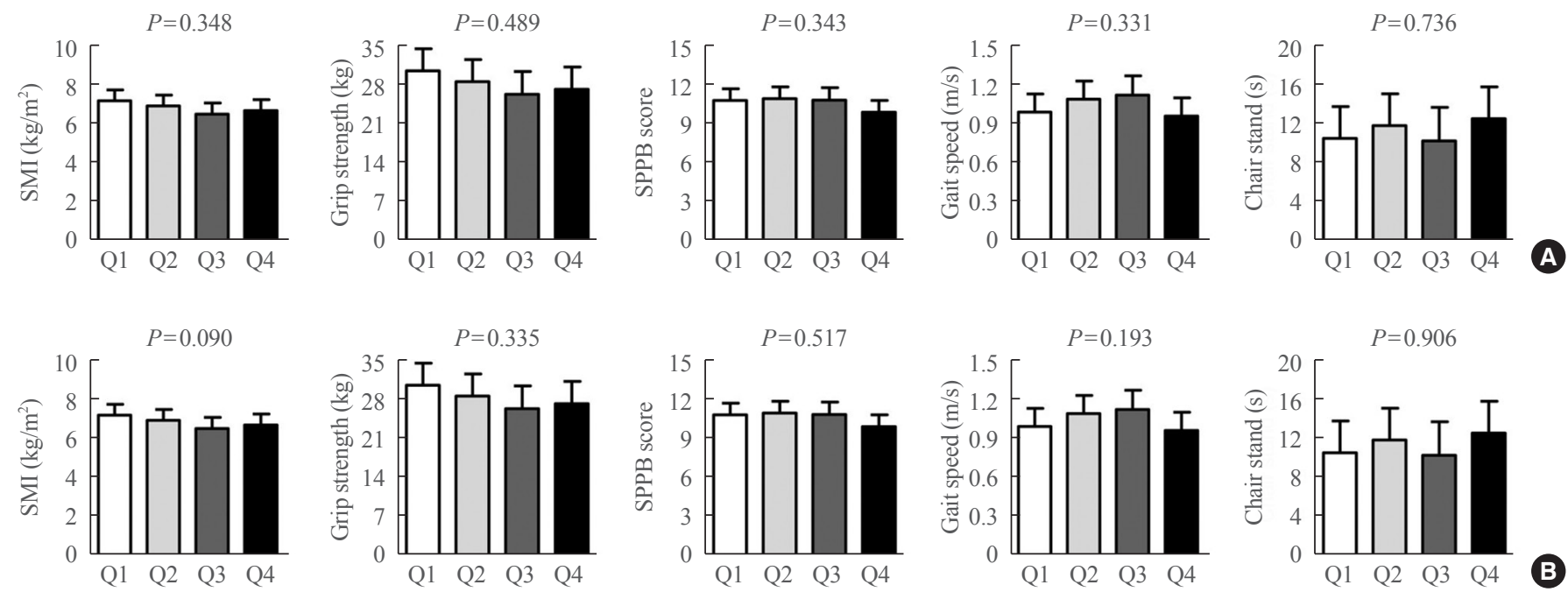

Fig. 4. Differences in the sarcopenia parameters based on the serum C-C motif chemokine ligand 11 (CCL11) quartiles (A) before and (B) after adjusting for sex, age, and body mass index (BMI). Analysis of covariance was used to generate and compare the estimated means with 95\% confidence intervals. Serum CCL11 quartiles: Q1, 46-115 pg/mL; Q2, 116-155 pg/mL; Q3, 156-195 pg/mL; and Q4, 196-327 pg/ $\mathrm{mL}$. SMI, skeletal muscle mass index; SPPB, short physical performance battery.

rogenesis, learning, memory, and bone remodeling [8,24]. Interestingly, clinical observations revealed that changes in one organ system, such as the brain, may occur alongside degenerative changes in other tissues, such as bone or muscle. Specifically, patients with cognitive dysfunction and Alzheimer's disease were more likely to experience loss of bone mass and muscle strength [37-39]. These observations suggest that CCL11 could function as a systemic factor related to a simultaneous physiological decline with aging and be also implicated in the pathogenesis of sarcopenia. However, contrary to expectations, recombinant CCL11 promoted the differentiation of mouse $\mathrm{C} 2 \mathrm{C} 12$ myoblasts into mature myotubes in our experiments. In other words, unlike in the brain or bone, CCL11 exhibited beneficial effects on muscle metabolism at least in vitro. According- ly, we surmised that CCL11 may not be the direct cause of sarcopenia, but rather its level could be increased with aging to overcome poor muscle health as a compensatory mechanism.

Notably, we found that the action of CCL11 on myogenesis was mediated via CCR5, not CCR3. CCL11 is a ligand for the following CCRs: CCR2, CCR3, and CCR5 [28,29]. Among these, CCL11 is known to bind to CCR3 with the highest affinity in several cell types, including eosinophils, basophils, and airway epithelial cells $[35,40]$. Accordingly, we opted to initially focus on the interaction between CCL11 and CCR3 in the muscle. However, CCR3 was not detected in muscle cells while the CCR3 inhibitor did not change the recombinant CCL11-induced myogenic differentiation (data not shown). On the other hand, CCR5 was strongly expressed in both myoblasts and myotubes, 
and CCR5 inhibitor completely reversed the myogenic differentiation stimulated by recombinant CCL11. Such results support the finding that CCR5 is the major receptor for CCL11 in skeletal muscle.

To expand on the experimental results revealing the beneficial effects of recombinant CCL11 on myotubes in vitro, we would like to elucidate its role in human muscle health. Because interventional trials cannot be conducted in humans without preclinical data confirming the safety verification, the roles of candidate factors in human muscle metabolism should be extrapolated from observational studies, which was the motivation for the present clinical study. However, an association between circulating CCL11 concentration and muscle mass, muscle strength, and physical performance was not recognized in older adults. Although the exact cause of this discrepancy could not be confirmed herein, we speculate that the physiologic CCL11 concentration in humans may not be high enough to exert the phenotypic changes expected. Specifically, CCL11 effectively promoted in vitro myogenic differentiation when administered at a dose of $10 \mathrm{nM}(84,000 \mathrm{pg} / \mathrm{mL})$, whereas the mean serum CCL11 level in all participants and the difference in mean serum CCL11 level between the lowest (Q1) and highest (Q4) quartiles were 159 and $165 \mathrm{pg} / \mathrm{mL}$, respectively. Because of the relatively low concentration of circulating CCL11 in humans, the clinical relevance of CCL11 for sarcopenia may not be observed. Furthermore, there is a chance that ligands for CCR5 other than CCL11 could bias the human results which were somewhat inconsistent with those of the in vitro experiments.

Since proteomics analyses by Villeda et al. [8] found that CCL11 concentrations were increased in the blood of humans and mice during normal aging, this molecule has been receiving increased attention as a potential pro-aging factor. In the present study, we recapitulated this positive association between chronological age and circulating CCL11 levels. However, although CCL11 is known to be produced in various types of cells including chondrocytes, keratinocytes, fibroblasts, eosinophils, epithelial cells, and endothelial cells [22,24,41], the specific mechanisms underlying age-related systemic increase of CCL11 remain unclear. To fully understand the biologic role of CCL11 in age-related diseases, this question needs to be addressed by additional research.

The major strength of this study is our assessment of all required parameters for clinical research on sarcopenia, including muscle mass, handgrip strength, SPPB, 5-time chair-stand test, and gait speed, which enhance the reliability of the results $[17,34]$. Moreover, we diagnosed sarcopenia by adopting Asian- specific cut-off points, because muscle phenotypes can vary according to cultural backgrounds, lifestyle, ethnicities, and body size [34]. Despite the above strengths, several limitations should be considered when interpreting the data. First, we could not examine the role of blood CCL11 in the prediction of sarcopenia due to the study's cross-sectional design. Second, our study population was exclusively Koreans. Thus, our results may not be applicable to other populations. Third, our bioelectrical impedance analysis methodology might not provide valid estimates of total body water when hydration states are altered (e.g., ascites, edema, and fluid loss), potentially leading to errors in the muscle mass assessments. Finally, uncontrolled factors that influence muscle metabolism and/or serum CCL11 level, such as 25 -hydroxyvitamin $\mathrm{D}$, may bias the observed findings.

In conclusion, although recombinant CCL11 stimulated in vitro myogenic differentiation, serum CCL11 concentration was not significantly different based on sarcopenia and the related parameters in older adults. Further animal interventional and clinical prospective studies are necessary to confirm the exact role of CCL11 in muscle metabolism.

\section{CONFLICTS OF INTEREST}

No potential conflict of interest relevant to this article was reported.

\section{ACKNOWLEDGMENTS}

This study was supported by grants from the National Research Foundation of Korea, funded by the South Korean government, MSIT (2019R1A2C2006527) and from the Korean Endocrine Society of EnM Research Award 2020.

\section{AUTHOR CONTRIBUTIONS}

Conception or design: D.A.K., S.J.P., J.H.P., B.J.K. Acquisition, analysis, or interpretation of data: D.A.K., J.Y.L., J.H.K., S.L., E.J., I.Y.J., H.W.J., B.J.K. Drafting the work or revising: D.A.K., S.J.P., J.H.P., B.J.K. Final approval of the manuscript: D.A.K., S.J.P., J.Y.L., J.H.K., S.L., E.J., I.Y.J., H.W.J., J.H.P., B.J.K.

\section{ORCID}

Da Ae Kim https://orcid.org/0000-0001-6137-1313

So Jeong Park https://orcid.org/0000-0003-0839-3016

Jin Hoon Park https://orcid.org/0000-0002-0903-3146 
Beom-Jun Kim https://orcid.org/0000-0001-8591-1759

\section{REFERENCES}

1. Moretto J, Girard C, Demougeot C. The role of arginase in aging: a systematic review. Exp Gerontol 2019;116:54-73.

2. Lopez-Otin C, Blasco MA, Partridge L, Serrano M, Kroemer G. The hallmarks of aging. Cell 2013;153:1194-217.

3. Hou Y, Dan X, Babbar M, Wei Y, Hasselbalch SG, Croteau $\mathrm{DL}$, et al. Ageing as a risk factor for neurodegenerative disease. Nat Rev Neurol 2019;15:565-81.

4. Niccoli T, Partridge L. Ageing as a risk factor for disease. Curr Biol 2012;22:R741-52.

5. Schosserer M, Grillari J, Wolfrum C, Scheideler M. Age-induced changes in white, brite, and brown adipose depots: a mini-review. Gerontology 2018;64:229-36.

6. Barja G. Towards a unified mechanistic theory of aging. Exp Gerontol 2019;124:110627.

7. Katsimpardi L, Litterman NK, Schein PA, Miller CM, Loffredo FS, Wojtkiewicz GR, et al. Vascular and neurogenic rejuvenation of the aging mouse brain by young systemic factors. Science 2014;344:630-4.

8. Villeda SA, Luo J, Mosher KI, Zou B, Britschgi M, Bieri G, et al. The ageing systemic milieu negatively regulates neurogenesis and cognitive function. Nature 2011;477:90-4.

9. Laviano A. Young blood. N Engl J Med 2014;371:573-5.

10. Villeda SA, Plambeck KE, Middeldorp J, Castellano JM, Mosher KI, Luo J, et al. Young blood reverses age-related impairments in cognitive function and synaptic plasticity in mice. Nat Med 2014;20:659-63.

11. Loffredo FS, Steinhauser ML, Jay SM, Gannon J, Pancoast JR, Yalamanchi P, et al. Growth differentiation factor 11 is a circulating factor that reverses age-related cardiac hypertrophy. Cell 2013;153:828-39.

12. Rebo J, Mehdipour M, Gathwala R, Causey K, Liu Y, Conboy MJ, et al. A single heterochronic blood exchange reveals rapid inhibition of multiple tissues by old blood. Nat Commun 2016;7:13363.

13. Kim BJ, Lee SH, Koh JM. Clinical insights into the kynurenine pathway in age-related diseases. Exp Gerontol 2020;130:110793.

14. Choi KM. Sarcopenia and sarcopenic obesity. Endocrinol Metab (Seoul) 2013;28:86-9.

15. Kim TN, Choi KM. Sarcopenia: definition, epidemiology, and pathophysiology. J Bone Metab 2013;20:1-10.

16. Kim G, Kim JH. Impact of skeletal muscle mass on meta- bolic health. Endocrinol Metab (Seoul) 2020;35:1-6.

17. Cruz-Jentoft AJ, Bahat G, Bauer J, Boirie Y, Bruyere O, Cederholm T, et al. Sarcopenia: revised European consensus on definition and diagnosis. Age Ageing 2019;48:16-31.

18. Cruz-Jentoft AJ, Sayer AA. Sarcopenia. Lancet 2019;393: 2636-46.

19. Dao T, Green AE, Kim YA, Bae SJ, Ha KT, Gariani K, et al. Sarcopenia and muscle aging: a brief overview. Endocrinol Metab (Seoul) 2020;35:716-32.

20. Lim HS, Park YH, Suh K, Yoo MH, Park HK, Kim HJ, et al. Association between sarcopenia, sarcopenic obesity, and chronic disease in Korean elderly. J Bone Metab 2018;25: 187-93.

21. Ponath PD, Qin S, Post TW, Wang J, Wu L, Gerard NP, et al. Molecular cloning and characterization of a human eotaxin receptor expressed selectively on eosinophils. J Exp Med 1996;183:2437-48.

22. Garcia-Zepeda EA, Rothenberg ME, Ownbey RT, Celestin J, Leder P, Luster AD. Human eotaxin is a specific chemoattractant for eosinophil cells and provides a new mechanism to explain tissue eosinophilia. Nat Med 1996;2:449-56.

23. Jose PJ, Griffiths-Johnson DA, Collins PD, Walsh DT, Moqbel R, Totty NF, et al. Eotaxin: a potent eosinophil chemoattractant cytokine detected in a guinea pig model of allergic airways inflammation. J Exp Med 1994;179:881-7.

24. Kindstedt E, Holm CK, Sulniute R, Martinez-Carrasco I, Lundmark R, Lundberg P. CCL11, a novel mediator of inflammatory bone resorption. Sci Rep 2017;7:5334.

25. Lee JY, Park SJ, Kim DA, Lee SH, Koh JM, Kim BJ. Muscle-derived lumican stimulates bone formation via integrin $\alpha 2 \beta 1$ and the downstream ERK signal. Front Cell Dev Biol 2020;8:565826.

26. Kim BJ, Lee YS, Lee SY, Baek WY, Choi YJ, Moon SA, et al. Osteoclast-secreted SLIT3 coordinates bone resorption and formation. J Clin Invest 2018;128:1429-41.

27. Park SJ, Lee JY, Lee SH, Koh JM, Kim BJ. SLIT2 inhibits osteoclastogenesis and bone resorption by suppression of Cdc42 activity. Biochem Biophys Res Commun 2019;514: 868-74.

28. Jang IY, Lee S, Kim JH, Lee E, Lee JY, Park SJ, et al. Lack of association between circulating apelin level and frailtyrelated functional parameters in older adults: a cross-sectional study. BMC Geriatr 2020;20:420.

29. Roberts HC, Denison HJ, Martin HJ, Patel HP, Syddall H, Cooper $\mathrm{C}$, et al. A review of the measurement of grip strength in clinical and epidemiological studies: towards a 
standardised approach. Age Ageing 2011;40:423-9.

30. Peel NM, Kuys SS, Klein K. Gait speed as a measure in geriatric assessment in clinical settings: a systematic review. J Gerontol A Biol Sci Med Sci 2013;68:39-46.

31. Jung HW, Roh H, Cho Y, Jeong J, Shin YS, Lim JY, et al. Validation of a multi-sensor-based kiosk for short physical performance battery. J Am Geriatr Soc 2019;67:2605-9.

32. Oh JH, Song S, Rhee H, Lee SH, Kim DY, Choe JC, et al. Normal reference plots for the bioelectrical impedance vector in healthy Korean adults. J Korean Med Sci 2019;34: e198.

33. Jang IY, Jung HW, Lee CK, Yu SS, Lee YS, Lee E. Comparisons of predictive values of sarcopenia with different muscle mass indices in Korean rural older adults: a longitudinal analysis of the Aging Study of PyeongChang Rural Area. Clin Interv Aging 2018;13:91-9.

34. Chen LK, Woo J, Assantachai P, Auyeung TW, Chou MY, Iijima K, et al. Asian working group for sarcopenia: 2019 consensus update on sarcopenia diagnosis and treatment. J Am Med Dir Assoc 2020;21:300-7

35. Kitaura M, Nakajima T, Imai T, Harada S, Combadiere C, Tiffany HL, et al. Molecular cloning of human eotaxin, an eosinophil-selective CC chemokine, and identification of a specific eosinophil eotaxin receptor, CC chemokine receptor 3. J Biol Chem 1996;271:7725-30.

36. Ogilvie P, Bardi G, Clark-Lewis I, Baggiolini M, Uguccioni M. Eotaxin is a natural antagonist for CCR2 and an agonist for CCR5. Blood 2001;97:1920-4.

37. Boyle PA, Buchman AS, Wilson RS, Leurgans SE, Bennett DA. Association of muscle strength with the risk of Alzheimer disease and the rate of cognitive decline in community-dwelling older persons. Arch Neurol 2009;66:1339-44.

38. Gray SL, Anderson ML, Hubbard RA, LaCroix A, Crane PK, McCormick W, et al. Frailty and incident dementia. J Gerontol A Biol Sci Med Sci 2013;68:1083-90.

39. Loskutova N, Honea RA, Vidoni ED, Brooks WM, Burns JM. Bone density and brain atrophy in early Alzheimer's disease. J Alzheimers Dis 2009; 18:777-85.

40. Ye J, Kohli LL, Stone MJ. Characterization of binding between the chemokine eotaxin and peptides derived from the chemokine receptor CCR3. J Biol Chem 2000;275:27250-7.

41. Alblowi J, Tian C, Siqueira MF, Kayal RA, McKenzie E, Behl Y, et al. Chemokine expression is upregulated in chondrocytes in diabetic fracture healing. Bone 2013;53:294300 . 